\title{
REVISÃO SISTEMÁTICA SOBRE IMPLICAÇÕES DO USO DE EMBALAGENS COM BISFENOL A - BPA PARA A SAÚDE HUMANA
}

\author{
Sofia Beatris Hoffmann ${ }^{1}$, Adriana Regina Bitello ${ }^{2}$
}

\begin{abstract}
Resumo: Esta revisão bibliográfica tem por objetivo investigar a associação entre a exposição ao Bisfenol A (BPA), presente em embalagens alimentares, e a ação deste sobre a saúde humana. Foi realizada revisão sistemática da literatura, consultando as bases de dados SCIELO (descritor: Bisfenol A), MEDLINEBirene (descritor: Bisfenol A) e PUBMED (descritor: Bisfenol A e toxicidade; exposição ao Bisfenol A) entre os anos 2005 e 2015. Como resultado, foram analisados 22 ensaios, estes demonstraram evidências de correlação entre disfunções endócrino-hormonais, obesidade e processos celulares e Bisfenol A, a partir de análises de sangue e urina. Conclui-se que os mecanismos da associação entre Bisfenol A e alterações endócrinas, hormonais e de processos celulares ainda não foram esclarecidos, mas os estudos têm evidenciado alterações na saúde humana relacionadas com BPA.
\end{abstract}

Palavras-chave: Bisfenol A. Recipientes alimentares. Metabolismo. Saúde humana.

\section{SYSTEMATIC REVIEW ON IMPLICATIONS FOR USE PACKAGING WITH BISPHENOL A - BPA HUMAN HEALTH}

\begin{abstract}
This literature review aims to investigate the association between exposure to bisphenol A (BPA), found in food packaging, and the action of this on human health. Systematic literature review was carried out by consulting the databases SCIELO (descriptor: Bisphenol A), MEDLINE-Birene (descriptor: Bisphenol A) and PubMed (descriptor: Bisphenol A and toxicity; exposure to Bisphenol A) between 2005 and 2015. As a result, 22 trials were analyzed, they showed evidence of correlation between endocrine and hormone dysfunction, obesity and cellular processes and Bisphenol A from blood and urine. We conclude that the association mechanisms between Bisphenol A and endocrine, hormonal and cellular processes change are still unclear, but studies have shown changes in human health from BPA.
\end{abstract}

Keywords: Bisphenol A. Food containers. Metabolism. Human health.

1 Química Industrial, Pós-Graduanda em Gestão em Segurança Alimentar e Nutricional-UNIVATES.

2 Nutricionista, Mestra em Biotecnologia - UNIVATES. 


\section{INTRODUÇÃO}

Bisfenol A (BPA) é o nome comum para 2,2- (4,4'-hidroxifenilico) propano, 4,4'-isopropilidenodifenol, ou 2,2'-bis (4-hidroxifenil) propano. Ele é utilizado como um intermediário na produção de resinas epóxi que são utilizadas no revestimento interno para latas de alimentos e bebidas para proteger os alimentos do contato direto com o metal. BPA pode migrar de latas com revestimento de epóxi em alimentos, especialmente em temperaturas elevadas (por exemplo, de hot-fill ou alimentos enlatados e processados termicamente (HEALTH CANADA, 2015). Também constitui plásticos policarbonatos que têm sido usados em mamadeiras, revestimentos de proteção de embalagens, recipientes para alimentos, água e afins e compósitos selantes em odontologia (CALAFAT et al., 2005).

Latas metálicas de alimentos e bebidas são protegidas contra ferrugem e corrosão pela aplicação de resinas epoxi como revestimento interno. A síntese de muitas dessas resinas requer a condensação de BPA com epicloridrina para criar BPA éter diglicidílico, quando a polimerização incompleta ocorre, BPA residual lixivia a partir da resina epoxi. Temperaturas elevadas e a exposição a ácido ou soluções básicas podem também aumentar a lixiviação de BPA de tintas e plásticos que revestem a embalagem, mesmo quando a polimerização completa ocorre, para dentro do alimento ou líquido nesta embalagem contido, tornando-o uma fonte de absorção de BPA ao consumidor (VANDENBERG et al., 2010). Podemos considerar uma fonte importante de exposição humana ao BPA a ingestão de alimentos e bebidas, os quais permanecem em contato com resinas epoxi ou plásticos de policarbonato (CARWILE et al., 2009). Lorber et al. (2015), em artigo de avaliação da exposição de adulto ao Bisfenol A por ingestão de alimentos, com ênfase em alimentos enlatados em dieta de exposição, constatou que a exposição ao BPA por ingestão de alimentos, principalmente, enlatados é superior à esperada, devido ao alto percentual de amostras positivas para concentrações de BPA.

As consequências da exposição ao BPA vêm sendo acompanhadas por pesquisadores, instituições de pesquisa e órgãos regulamentadores. Estudos em animais, conforme publicação de Vrooman et al. (2015), demonstram que o BPA e outros produtos químicos, que atuam como desreguladores, têm ocasionado efeitos negativos sobre uma grande variedade de processos fisiológicos, incluindo a reprodução. Neste estudo em experimentos realizados com ratas, a exposição de BPA aumentou erros meióticos, resultando na produção de ovos cromossomicamente anormais.

Desreguladores endócrinos, como BPA, podem imitar hormônios ou parte destes, que ocorrem naturalmente no corpo como estrógenos (hormônio sexual feminino), andrógenos (hormônio sexual masculino) e hormônios da tireoide, potencialmente, produzindo superestimulação e, com isso, ligam-se a um receptor em uma célula e bloqueiam a ligação endócrino-hormonal (NIEHS, 2015).

O BPA também tem atraído a atenção de agências reguladoras e de cientistas, em dezenas de países, por suas propriedades estrogênicas in vitro e in vivo e a interferência 
no papel do estrogênio, na conservação e na regulação fisiológica e fisiopatológica humana e animal (VANDENBERG et al., 2010).

Desta forma, a presente revisão bibliográfica, direcionada a área da saúde coletiva e tecnologia de produção, visa reunir os principais achados escritos relativos a alterações endócrinas, hormonais e fisiológicas em seres humanos associados a presença de Bisfenol A no corpo, proveniente de embalagens alimentares. Torna-se mais significativa visto que não são encontrados, na literatura, estudos que possam fornecer dados sobre estas associações. Também, devido à falta de dados clínicos, desta associação em seres humanos, no Brasil.

\section{MÉTODO}

Estudo de revisão sistemática da literatura, para tal, foram consultadas as bases de dados SciELO, MEDLINE-Birene e PUBMED e selecionados artigos científicos publicados em Inglês, Português e Espanhol, buscando ensaios clínicos publicados nos últimos dez anos (2005 a 2015). Os descritores utilizados para a pesquisa de artigos nas bases foram os seguintes: Bisfenol A e Toxicidade, Exposição ao Bisfenol A, Disruptores Endócrinos e Bisfenol A e seus equivalentes em língua inglesa. Com a busca identificou-se o número de 820 artigos, dentre os quais foram selecionados 22 de acordo com o objetivo deste estudo. Num primeiro momento, analisou-se o título e após o resumo. Além destes, foram incluídas mais três referências devido à relevância para o estudo, na conceituação e aplicação do BPA.

\section{RESULTADOS E DISCUSSÃO}

\subsection{Presença de BPA em Alimentos, Embalagens e Humanos}

O BPA é muito utilizado em embalagens alimentares e avaliar a taxa de transferência deste produto para alimentos e água e as interações deste com a saúde humana torna-se necessária. Neste sentido, nos Estados Unidos da América (USA), um estudo com 77 estudantes universitários foi realizado para comparar as concentrações de BPA urinário, estes estudos demonstraram que ao consumir água em embalagens de policarbonato as concentrações urinárias de BPA aumentaram (CARWILE et al., 2009). Estes níveis de concentração de BPA em urina apresentaram-se ainda maiores, principalmente em mulheres, quando a água consumida encontrava-se em recipientes de policarbonatos e estavam submetidos a temperaturas mais elevadas, o que ocorre durante o verão, quando tem-se aumento na temperatura e maior incidência de raios ultravioletas (UV) (MAKRIS et al., 2013). Estes fatores colaboram para maior lixiviação de BPA da embalagem para a água nela contida. 
Em um estudo com alimentos, foram coletadas 204 amostras de alimentos frescos, enlatados e congelados e BPA foi positivo em $73 \%$ das amostras de alimentos enlatados e 7\% em alimentos não enlatados (LORBER et al., 2015). Em outro estudo, realizado no Canadá com amostras de alimentos enlatados, das amostras coletadas, 55 (36\%) apresentaram migração específica do composto, sendo que a concentração de BPA para produtos como conserva de peixes (106 ng.g-1), conserva de milho (83,7 ng.g-1) e sopas enlatadas (22,2 - 44,4 ng.g-1) apresentou-se muito acima da especificação limite de migração (0,6 ng.g-1) (CAO et al., 2011).

Ainda relacionando consumo de alimentos e contaminação com BPA, podese citar estudo realizado com voluntários que consumiram sopas frescas e sopas enlatadas, o estudo detectou concentrações de BPA urinário em $77 \%$ das amostras cuja alimentação do participante havia sido baseada em sopas frescas e $100 \%$ para as amostras de consumidores de sopas enlatadas (CARWILE et al., 2011). Constatando, com este estudo, a presença contínua de BPA em alimentos e mais intensa quando o recipiente de conservação destes alimentos trata-se de recipiente de policarbonato ou lata com camada de proteção.

\subsection{Alterações na Saúde Associadas ao BPA}

Segundo o Institute Experimental Endocrinology and Oncology (IEOS), da Universidade de Nápoles, em um estudo (SAVASTANO et al., 2015) realizado com 76 de 139 adultos do sexo masculino, ambientalmente expostos ao BPA, matriculados em um inquérito de saúde de rotina. Desses 76, 24 indivíduos (32\%) apresentaram circunferência da cintura $(\mathrm{CC})>102$ centímetros, 36 (47\%) indivíduos apresentaram elevada glicemia em jejum e $24(32 \%)$ indivíduos tinham resistência à insulina. BPA e os níveis de citocinas pró-inflamatórias foram significativamente maiores em indivíduos com adiposidade visceral $(\mathrm{CC}>102 \mathrm{~cm})$. $\mathrm{O}$ estudo conclui que níveis plasmáticos detectáveis de BPA foram encontrados na população estudada e a associação estrita entre BPA, circunferência de cintura e marcadores inflamatórios, amparam ainda mais o papel BPA na obesidade visceral relacionada com baixo grau de inflamação crônica.

Quanto a estudo relacionando concentrações de BPA e diabetes (KIM; PARK, 2013), no Irã, os resultados apontaram para conclusão de que os níveis urinários de Bisfenol A encontram-se associados com diabetes, independente de fatores de risco tradicionais, sendo necessários estudos para esclarecer os mecanismos destas associações. Participaram deste estudo 239 pessoas adultas, com média de idade de 51,65 anos, destas, 119 tinham diabetes mellitus tipo 2. A classificação foi aplicada com base na mediana de BPA urinário em dois grupos ( $\mathrm{a}>0,85 \mathrm{ug} / \mathrm{L} \leq 0)$. Na Tailândia, foi realizado um estudo transversal representativo da população (AEKPLAKORN; CHAILURKIT; ONGPHIPHADHANAKUL, 2015), em 2009, incluindo um total de 21.960 pessoas. Destas, foram selecionadas aleatoriamente 2.581 amostras de soro, nas quais se detectou concentrações de BPA em 2.135 amostras $(78,1 \%)$, sendo que a concentração média geométrica ficou em $0,34 \mathrm{ng} / \mathrm{mL}$ de BPA. Os níveis séricos de 
BPA foram significativamente maiores entre aqueles com diabetes quando comparados com indivíduos normoglicêmicos, sendo que, após ajustes de cálculos, constatou-se maior fator de associação para homens do que mulheres.

No Hospital da Universidade de Nanchang, China, o estudo realizado com 88 pacientes (XIONG et al., 2015), teve como objetivo avaliar as concentrações séricas de BPA em pacientes com cardiomiopatia dilatada (CMD), bem como a associação entre os níveis séricos BPA e outros parâmetros hormonais, como Testosterona total (T), Globulina Transportadora de Hormônio Sexual (SHBG), Estradiol (E2) e o Índice de Andrógeno Livre (IAL), em pacientes com CMD e grupo controle saudável. Os resultados demonstraram que os níveis séricos de BPA foram mais elevados em pacientes com CMD ao comparar com os controles saudáveis $(6,9 \pm 2,7 \mathrm{ng} / \mathrm{mL}$ comparativamente com 3,8 $\pm 1,9 \mathrm{ng} / \mathrm{mL}, \mathrm{p}<0,001)$. Em contrapartida, os níveis de IAL e T diminuíram. Já SHBG apresentou associação positiva com BPA. O estudo concluiu que a desordem hormonal induzida pela exposição ao BPA pode ser um fator ambiental na patologia de CMD.

Nos EUA, estudo realizado utilizando Banco de dados NHANES 2003/2006 (MELZER et al., 2010), valeu-se do número $\mathrm{n}=1.455$ amostras $(2003 / 2004)$ e $\mathrm{n}=1.493$ amostras (2005/2006), com idades entre 18 - 74 anos, representativos da população geral adulta dos Estados Unidos. As concentrações de BPA na urina da população da amostragem NHANES 2005/06 foram mais baixas quando comparadas aos dados de 2003/04, mas os valores replicam as conclusões anteriores: as concentrações urinárias mais elevadas de Bisfenol A estão associadas com um aumento da prevalência de doença cardíaca coronária. Não houve associação global com concentrações de gamaglutamiltransferase, mas as associações reunidas com fosfatase alcalina e da lactato desidrogenase permaneceram significativas.

Ao tratar especificamente da saúde de homens, na Itália, um estudo sobre exposição ao Bisfenol A associada, in vivo, com Expressão Estrogênica em Adultos (MELZER et al., 2011), avaliou amostras de leucócitos de sangue periférico de 96 homens adultos, valendo-se do estudo populacional InCHIANTI, sendo examinadas as expressões de seis receptores estrogênicos e genes de receptores de andrógenos. Em modelos ajustados por idade, constataram-se associações positivas entre as concentrações de BPA mais elevadas e ESR2 [2 receptor (ER beta) estrogênio] e ESRRA (alfa estrogênio relacionado com o receptor). Em $65 \%$ dos indivíduos pertencentes ao tercil superior de concentração de BPA, a expressão de ESR2 do gene em leucócitos do sangue periférico foi significativamente maior quando comparado ao tercil inferior. $\mathrm{O}$ significado clínico destes resultados ainda não é conhecido, mas esta ativação em humanos evidencia que BPA é suscetível a atuação como xenoestrogênio em adultos. $\mathrm{O}$ estudo conclui que há uma necessidade de replicação e acompanhamento científico, no caso da análise da relação entre as mudanças de expressão do gene e a expressão da proteína e os efeitos da exposição de BPA em uma ampla gama de tecidos alvo regulados por estrogênios. 
Já ao tratar sobre saúde da mulher e reprodução, variados estudos têm sido desenvolvidos, um destes foi conduzido com ovários de 6 fetos euplóides (BRIEÑOENRIQUEZ et al., 2012). O estudo dedicou-se a caracterizar a expressão gênica de oócitos fetais humanos em cultura, bem como avaliar o efeito do BPA nesse oócitos cultivados. A investigação focou a indução do BPA sobre a expressão de genes envolvidos, visto que o composto tem sido relacionado a anomalias meióticas, aborto espontâneo recorrente, cariótipo anormal, a diminuição da sobrevivência do oócito, o atraso na progressão da meiose e uma elevada taxa do gene MLH1 in vitro. Os resultados obtidos demonstram que Bisfenol A atua sobre a expressão de genes (Spo11, $\mathrm{H} 2 \mathrm{AX}$, e Rpa, Blm) em culturas ovarianas fetais humanas, sendo que esses genes estão envolvidos na ruptura de fita dupla (RFD), sinalização e reparação durante a meiose, e alguns são compartilhados com a mitose (H2AX, Rpa e Blm). Os efeitos de BPA no ovário fetal apresentam-se como em um órgão, sendo necessário lembrar que, no final da prófase da meiose, os oócitos estabelecem uma relação próxima com as células do estroma do ovário para formar os folículos primordiais, que são a fonte de precursores de gametas na fêmea. Assim, a atuação do BPA no estroma celular do tecido necessita ser considerado ao estudar o efeito deste na função ovariana.

Outra pesquisa relacionada com reprodução humana foi realizada na China, para investigar a relação concentração de BPA e abortos recorrentes (AR) (SHEN et al., 2015). O grupo foi formado por 102 pacientes com abortos recorrentes e 162 controles. Os níveis de BPA urinário, sem ajuste de creatinina, nas pacientes $\mathrm{AR}$ e controles foram $1,66 \pm 3,69 \mathrm{ng} / \mathrm{mL}$ e $0,58 \pm 1,07 \mathrm{ng} / \mathrm{mL}$, respectivamente, e com correção 0,98 $\pm 2,67 \mu \mathrm{g} / \mathrm{g}$ Creatinina e $0,40 \pm 0,77 \mu \mathrm{g} / \mathrm{g}$ Creatinina. Constatou-se que o nível de BPA em paciente AR apresentou-se mais elevado comparando com paciente controle, sendo associado o aumento do risco de aborto com concentração de BPA, independentemente da data do aborto.

O estudo realizado por Aghajanova e Giudice (2011) avaliou os efeitos do Bisfenol A em fibroblastos do estroma endometrial (FEE) e a diferenciação e expressão de genes envolvidos no metabolismo do estrogênio humano. Para isso foram coletados, em biopsia, endométrios de oito indivíduos e submetidos a altas doses de BPA. Os resultados apresentaram significativas alterações, dentre elas, indução de proteínas, alteração na conversão estradiol/estrona e efeito do receptor de estrogênio sobre a expressão do gene das células tratadas, associando BPA a desregulação endócrina em função do FEE humano e expressão gênica.

Referente à desregulação endócrina, um estudo sobre a associação de BPA e câncer de mama foi realizado nos EUA com linha de células MCF7 (cancro da mama humano, ER-positivo) (BHAN et al., 2014), sendo investigada a expressão HOTAIR, gene chave no silenciamento e regulado transcricionalmente pelo estradiol. Os resultados demonstram que BPA e Dietilestilbestrol (DES) podem induzir a expressão do promotor HOTAIR em células humanas de câncer de mama (MCF-7) em cultura e, também, in vivo nas glândulas mamárias de ratos. Em síntese, a exposição ao BPA 
e DES altera a programação epigenética dos promotores HOTAIR, ocasionando disfunção endócrina in vitro e in vivo.

O potencial de inibição de BPA em relação a alguns medicamentos tem sido foco de estudo na China (LI et al., 2014), investigando o potencial de inibição de BPA sobre microssomas do fígado humano (MFH) no sentido da catálise glucuronidação hepática da zidovudina (AZT). Os resultados do estudo demonstraram que BPA exerce inibição competitiva para a glucuronidação do AZT, sendo possível que BPA atue inibindo a atividade de outra isoforma, glucuronosiltransferase (UGT1A6), significativa para a utilização da serotonina (5-HT) e 4-metilumbeliferona (4-MU). Estes resultados indicam que a exposição ao BPA afeta a eliminação metabólica de algumas drogas clínicas devido a influência sobre a atividade de isoformas UGT.

\subsection{Alterações na saúde de crianças associadas ao BPA}

Concentrações de BPA foram detectadas em mais de $97 \%$ das gestantes (mediana: 2,0 $\mu \mathrm{g} / \mathrm{L}$ ) e na infância (mediana: 4,1 $\mu \mathrm{g} / \mathrm{L}$ ), em amostras de urina (BRAUN et al., 2011). Por este estudo, a elevação em 10 vezes na concentração de BPA gestacional foi associada com o comportamento mais ansioso e deprimido, menor controle emocional e inibição nas crianças. Concluindo que a exposição ao BPA gestacional afeta domínios comportamentais e emocionais de regulação aos três anos de idade, especialmente entre as meninas. Já em outro estudo (HARLEY et al., 2013), baseado nas concentrações de BPA urinário pré-natal, verificou associação de BPA com comportamento de internalização, desatenção e hiperatividade em meninos e meninas em idade sete anos. Sendo que para meninos constatou-se aumento de problemas de internalização, incluindo ansiedade e depressão. Em meninas com sete anos de idade, maiores concentrações de BPA urinários foram associados com o aumento de comportamentos de externalização, incluindo problemas de conduta. Assim, exposição ao BPA, já no início da vida, tem sido associada com problemas de comportamento, incluindo ansiedade, depressão e hiperatividade em crianças, considerando que informações adicionais sobre o calendário de exposição e diferenças entre os sexos são necessárias.

Ainda avaliando associações entre concentrações de BPA em soro em crianças, um total de 251 crianças do sexo feminino (com idade entre quatro meses a dois anos) e 33 indivíduos controle saudável participaram de um estudo (CHEN et al., 2015) que avaliou a telarca precoce. A concentração de BPA em soro no grupo com telarca prematura apresentou-se significativamente mais elevada do que no grupo de controle. Entretanto, não houve correlação entre BPA e idade e nível, mas constatou-se que a concentração de BPA em soro caiu com o aumento da idade. Além disso, os níveis séricos de BPA eram marcadamente maiores em lactantes com idade entre quatro meses a dois anos de idade.

A associação entre BPA e obesidade em adultos e crianças tem sido estudada na China, onde um estudo com 1.326 crianças entre quatro e doze anos (LI et al., 2013) 
coletou medidas antropométricas e urina para mensuração de BPA de $98 \%$ dos alunos elegíveis. Para 90\% das meninas com idade de nove a doze anos, constatou-se relação dose-resposta com o aumento do nível de BPA na urina, associada com aumento do risco de excesso de peso. O estudo sugere que o BPA pode ser um potencial fator de obesidade de origem ambiental, contribuindo para a epidemia mundial de obesidade. Nos EUA, foi examinada a associação entre níveis crescentes de BPA urinário e obesidade em crianças de seis a dezoito anos participantes do Estudo NHANES 20032004, 2005-2006 e 2007-2008 (BHANDARI; XIAO; SHANKAR, 2013). O Estudo apresentou associação positiva entre o aumento dos níveis de BPA na urina e obesidade.

\section{CONCLUSÃO}

A revisão de artigos científicos possibilitou sistematizar informações específicas quanto a associação entre a presença de BPA no organismo humano, adulto e criança, e alterações em processos endócrinos, hormonais e fisiológicos baseados em estudos com humanos.

Da mesma forma, permitiu compilar dados sobre a presença de BPA em embalagens de alimentos, de água e afins e sobre a transferência de BPA das embalagens para os alimentos e afins nelas contidos, revelando atuação como fonte de contaminação para adultos e crianças e trazendo preocupação, pois estas embalagens estão amplamente difundidas em todos os segmentos sociais, geográficos e faixas etárias.

A associação de níveis elevados de BPA, presentes no organismo, com disfunções reprodutivas, cardíacas, endócrinas e cerebrais, assim como alterações no metabolismo de absorção de medicamentos foi descrita por diferentes estudos, porém, em alguns estudos, não foi possível apontar relação estatisticamente significativa dos diagnósticos das alterações citadas com a presença de BPA no organismo, permanecendo ainda dúvidas sobre a relação causa-efeito de concentrações elevadas de BPA no organismo humano.

A realização de novos estudos clínicos, envolvendo novas populações, poderá contribuir para obtenção de maior número de informações e permitir que futuras abordagens terapêuticas possam ser melhor direcionadas. Da mesma forma, são fundamentais para fomentar a substituição do BPA em embalagens para alimentos e afins, a fim de evitar a contaminação do organismo pelo mesmo e consequentemente interações que afetem a saúde.

\section{REFERÊNCIAS}

AEKPLAKOM, Wichai; CHAILURKIT, La-or; ONGPHIPHADHANAKUL, Boonsong. Relationship of serum bisphenol A with diabetes in the Thai population, National Health Examination Survey IV, 2009. J Diabetes, 7 (2): 240-249, Mar. 2015. Disponível 
em: http://www.ncbi.nlm.nih.gov/pubmed/24720399. Acesso em: 20 Jun. 2015. doi: 10.1111/1753-0407.12159.

AGHAJANOVA, Lusine; GIUDICE, Linda C. Effect of bisphenol A on human endometrial stromal fibroblasts in vivo. Reprod. Biomed. Online, 22(23): 249-56, Mar. 2011. Disponível em: http://www.ncbi.nlm.nih.gov/pubmed/21273127. Acesso em: 20 Jul. 2015. doi: 10.1016/j. rbmo.2010.12.007

BHAN, Arunoday; HUSSAIN, Imran; ANSARI, Khairul I.; BOBZEAN, Samara A. M.; PERROTTI, Linda I.; MANDAL, Subhrangsu S. Bisphenol-A and diethylstilbestrol exposure induces the expression of breast cancer associated long noncoding RNA HOTAIR in vitro and in vivo. J Steroid Biochem Mol Biol., 141: 160-170, May. 2014. Disponível em: http://www.ncbi.nlm.nih.gov/pubmed/24533973. Acesso em: 20 Jul. 2015. doi: 10.1016/j. jsbmb.2014.02.002

BHANDARI, Ruchi; XIAO, Jie; SHANKAR, Anoop. Urinary Bisphenol A and Obesity in US Children. Am J Epidemiol., 177(11): 1263-1270, Jun. 2013. Disponível em: http://www. ncbi.nlm.nih.gov/pubmed/23558351. Acesso em: 20 Jul. 2015. doi: 10.1093/aje/kws391

BRAUN, Joe M.; KALKBRENNER, Amy E.; CALAFAT, Antonia M.; YOLTON, Kimberly; Ye, Xiaoyun; DIETRICH, Kim N.; LANPHEAR, Bruce P. Impact of earlylife bisphenol A exposure on behavior and executive function in children. Pediatrics, 128 (5): 873-882, Nov. 2011. Disponível em: http:/www.ncbi.nlm.nih.gov/pmc/articles/ PMC3208956/. Acesso em: 20 Jul. 2015. doi: 10.1542/peds.2011-1335.

BRIEÑO-ENRIQUEZ, M.A.; REIG-VIADER, R.; CABERO, L.; TORAN, N.; MARTINEZ, F.; ROIG, I.; GARCIA CALDÉS, M. Gene expression. Is altered after Bisphenol A exposure in human fetal oocytes in vitro. Molecular Human Reproduction, 18(14): 171-83, Apr. 2012. Disponível em: http://www.ncbi.nlm.nih.gov/pubmed/22121209. Acesso em: 21 Jul. 2015. doi: 10.1093/molehr/gar074

CALAFAT, Antonia M.; KUKLENYIK, Zsuzsanna; REIDY, John A.; CAUDILL, Samuel P.; EKONG, John; NEEDHAM, Larry L. Urinary Concentrations of Bisphenol A and 4-Nonylphenol in a Human Reference Population. Environ Health Perspect, 113(4): 391395, Apr. 2005. Disponível em: http://www.ncbi.nlm.nih.gov/pmc/articles/PMC1278476/. Acesso em: 18 Jul. 2015. doi: 10.1289/ehp.7534

CAO, X.L.; PEREZ-LOCAS, C.; DUFRESNE, G.; CLEMENT, G.; POPOVIC, S.; BERALDIN, F.; DABEKA, R.W.; FEELEY, M. Concentrations of bisphenol A in the composite food samples from the 2008 Canadian total diet study in Quebec City and dietary intake estimates. Food Addit Contam Part A Chem Anal Control Expo Risk Assess., 28(6): 791-8, Jun. 2011. Disponível em: http://www.ncbi.nlm.nih.gov/pubmed/21623504. Acesso em: 10 Jul. 2015. doi: 10.1080/19440049.2010.513015

CARWILE, Jenny L.; LUU, Henry T.; BASSETT, Laura S.; DRISCOLL, Daniel A.; YUAN, Caterina; CHANG, Jennifer Y.; YE, Xiaoyun; CALAFAT, Antonia M.; MICHELS, Karin B. Polycarbonate Bottle Use and Urinary Bisphenol A Concentrations.

Environ Health Perspect., 117(9): 1368-72, Sep. 2009. Disponível em: http://www.ncbi.nlm. nih.gov/pubmed/19750099. Acesso em: 12 Jul. 2015. doi: 10.1289/ehp.0900604

CARWILE, Jenny L.; YE, Xiaoyun; ZHOU, Xiaoliu; CALAFAT, Antonia M.;

MICHELS, Karin B. Canned Soup Consumption and Urinary bisphenol A: A Randomized 
Crossover Trial. JAMA, 306(20): 2218-2220, Nov. 2011. Disponível em: http://www.ncbi. nlm.nih.gov/pubmed/22110104. Acesso em: 21 Jul. 2015. doi: 10.1001/jama.2011.1721.

CHEN, Lian-Hui; SHI, Jian-rong; FANG, Yan-Lian; LIANG, Li; CHEN, Wan-Qin; CHEN, Xiao-Zhen. Serum bisphenol A concentration and premature thelarche in female infants aged 4-month to 2-year. The Indian Journal of Pediatrics, Volume 82, Issue 3, pp 221-224, Mar. 2015. Disponível em: http://www.ncbi.nlm.nih.gov/pubmed/25120062. Acesso em: 20 Aug. 2015. doi: 10.1007/s12098-014-1548-7

HARLEY, Kim G.; GUNIER, Robert B.; KOGUT, Katherine; JOHNSON, Caroline; BRADMAN, Asa; CALAFAT, Antonia M.; ESKENAZI, Brenda. Prenatal and early childhood bisphenol A concentrations and behavior in school-aged children. Environ. Res., 126: 43-50, Out. 2013. Disponível em: http://www.ncbi.nlm.nih.gov/pubmed/23870093. Acesso em: 20 Ago. 2015. doi: 10.1016/j.envres.2013.06.004

\section{HEALTH CANADA. Survey of Bisphenol A in Soft Drink and Beer Products from}

Canadian Markets. Bureau of Chemical Safety Food Directorate Health Products and Food Branch. Disponível em: http://publications.gc.ca/collections/collection_2011/sc-hc/H164125-2010-eng.pdf. Acesso em: 10 jul. 2015.

KIM, K.; PARK, H. Association between urinary concentrations of bisphenol A and type 2 diabetes in Korean adults: A population based cross-sectional study. Int. J. Hyg. Environ. Health, 216, 467-471, Jul. 2013. Disponível em: http:/www.ncbi.nlm.nih.gov/ pubmed/22921714. Acesso em: 30 Ago. 2015. doi: 10.1016/j.ijheh.2012.07.007

LI, De-Kun; MIAO, Maohua; ZHOU, ZhiJun; WU, Chunhua; SHI, Huijing; LIU, Xiaoqin; WANG, Siqi; YUAN, Wei. Urine Bisphenol-A Level in Relation to Obesity and Overweight in School-Age Children. PLoS ONE, 8(6): e65399, Jun. 2013. Disponível em: http://journals.plos.org/plosone/article?id=10.1371/journal.pone.0065399. Acesso em: 23 Ago. 2015. doi: 10.1371/journal.pone.0065399

LI, Wei-Ping; WANG, Yu-Feng; GAO, Jian; YU, Ming-Lian; YU, Yan-Yang; YAO, Yuan-Qing. In vitro evidence for endocrine-disrupting chemical (EDC)'s inhibition of drug metabolism. Afr Health Sci, 14(1): 185-188, Mar. 2014. Disponível em: http://www.ncbi. nlm.nih.gov/pubmed/26060477. Acesso em: 23 Ago. 2015. doi: 10.4314/ahs.v14i1.28

LORBER, Matthew; SCHECTER, Arnold; PAEPKE, Olaf; SHROPSHIRE, William; CHRISTENSEN, Krista; BIMBAUM, Linda. Exposure assessment of adult intake of bisphenol A (BPA) with emphasis on canned food dietary exposures. Environ Int., 77:55-62, Apr. 2015. Disponível em: http://www.ncbi.nlm.nih.gov/pubmed/25645382. Acesso em: 28 Jul. 2015. doi: 10.1016/j.envint.2015.01.008

MAKRIS, K.C.; ANDRA, S.S.; JIA, A.; HERRICK, L.; CHRISTOPHI, C.A.; SNYDER, S.A.; HAUSER, R. Association between water consumption from polycarbonate containers and bisphenol A intake during harsh environmental conditions in summer.

Environ Sci Technol., 2;47(7): 3333-43, Apr. 2013. Disponível em: http://www.ncbi.nlm. nih.gov/pubmed/23448553. Acesso em: 23 Ago. 2015. doi: 10.1021/es304038k

MELZER, David; RICE, Neil E.; LEWIS, Ceri; HENLEY, William E.; GALLOWAY, Tamara S. Association of Urinary Bisphenol A Concentration with Heart Disease: Evidence from NHANES 2003/2006. PLoS One, 5(1): e8673, Jan. 2010. Disponível em: http:// 
journals.plos.org/plosone/article?id=10.1371/journal.pone.0008673. Acesso em: 28 Ago. 2015. doi: 10.1371/journal.pone.0008673

MELZER, David; HARRIES, Lorna; CIPELLI, Riccardo; HENLEY, William; MONEY, Cathryn; McCORMACK, Paul; YOUNG, Anita; GURALNIK, Jack; FERRUCCI, Luigi; BANDINELLI, Stefania; CORSI, Anna Maria; GALLOWAY, Tamara. Bisphenol A Exposure Is Associated with in Vivo Estrogenic Gene Expression in Adults. Environ Health Perspect., 19(12): 1788-1793, Dec. 2011. Disponível em: http://www.ncbi.nlm.nih.gov/ pubmed/21831745. Acesso em: 10 Ago. 2015. doi: 10.1289/ehp.1103809

NATIONAL INSTITUTE OF ENVIRONMENTAL HEALTH SCIENCES.

Endocrine Disruptors. Disponível em: http://www.niehs.nih.gov/health/topics/agents/ endocrine/. Acesso em: 11 Jul. 2015.

SAVASTANO, Silvia; TARANTINO, Giovanni; D'ESPOSITO, Vittoria; PASSARETTI, Federica; CABARO, Serena; LIOTTI, Antonietta; LIGUORO, Domenico; PERRUOLO, Giuseppe; ARIEMMA, Fabiana; FINELLI, Carmine; BEGUINOT, Francesco; FORMISANO, Pietro; VALENTINO, Rossella. Bisphenol-A plasma levels are related to inflammatory markers, visceral obesity and insulin-resistance: a cross-sectional study on adult male population. Journal Translational Medicine, 13: 169, 2015. Disponível em: http:// www.translational-medicine.com/content/13/1/169. Acesso em: 20 Ago. 2015. doi: 10.1186/ s12967-015-0532-y

SHEN, Yueping; ZHENG, Yanmin; JIANG, Jingting; LIU, Yinmei; LUO, Xiaoming; SHEN, Zongji; CHEN, Xin; WANG, Yan; DAI, Yiheng; ZHAO, Jing; LIANG, Hong; CHEN, Aimin; YUAN, Wei. Higher Urinary Bisphenol A Concentration Is Associated with Unexplained Recurrent Miscarriage Risk: Evidence from a Case Control Study in Eastern China. PLoS One, 10(5): e0127886, May. 2015. Disponível em: http://www.ncbi. nlm.nih.gov/pmc/articles/PMC4444137/. Acesso em: 23 Ago. 2015. doi: 10.1371/journal. pone. 0127886

VANDENBERG, Laura N.; CHAHOUD, Ibrahim; HEINDEL, Jerrold J.; PADMANABHAN, Vasantha; PAUMGARTTEN, Francisco J.R.; SCHOENFELDER, Gilbert. Urinary, Circulating, and Tissue Biomonitoring Studies Indicate Widespread Exposure to Bisphenol A. Environ Health Perspect., 118(8): 1055-70, Aug. 2010. Disponível em: http://www.ncbi.nlm.nih.gov/pubmed/20338858. Acesso em: 23 Ago. 2015. doi: 10.1289/ehp.0901716

VROOMAN, Lisa A.; OATLEY, Jon M.; GRISWOLD, Jodi E.; HASSOLD, Terry J.; HUNT, Patricia A. Estrogenic Exposure Alters the Spermatogonial Stem Cells in the Developing Testis, Permanently Reducing Crossover Levels in the Adult. PLoS Genet., 11(1): e1004949, Jan. 2015. Disponível em: http://journals.plos.org/plosgenetics/ article?id=10.1371/journal.pgen.1004949. Acesso em: 20 Jul. 2015. doi: 10.1371/journal. pgen.1004949

XIONG, Qinmei; LIU, Xiao; SHEN, Yang; YU, Peng; CHEN, Sisi; HU, Jinzhu; YU, Jianhua; LI, Juxiang; WANG, Hong-Sheng; CHENG, Xiaoshu; HONG, Kui. Elevated Serum Bisphenol A Level in Patients with Dilated Cardiomyopathy. Int J Environ Res Public Health, 12(5): 5329-5337, May 2015. Disponível em: http://www.ncbi.nlm.nih.gov/ pubmed/25996886. Acesso em 20 Jul. 2015. doi: 10.3390/ijerph120505329 